\title{
Supporting Information \\ Single-Molecule Localization Microscopy with the Fluorescence-Activating and Absorption-Shifting Tag (FAST) System
}

\author{
Elizabeth M. Smith ${ }^{\dagger}$, Arnaud Gautier", and Elias M. Puchner*, ${ }^{*}$. \\ †School of Physics and Astronomy, University of Minnesota, Minneapolis, MN 55455 \\ \# PASTEUR, Département de Chimie, École Normale Supérieure, PSL University, Sorbonne \\ Université, CNRS, 75005 Paris, France
}

\section{Table of Contents}

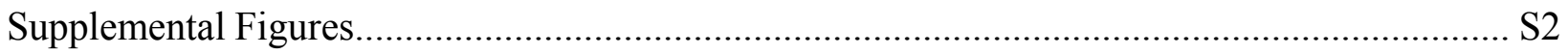

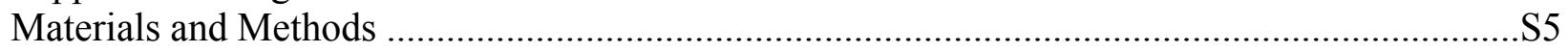

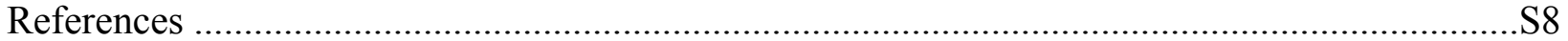

\section{Supplemental Figures}

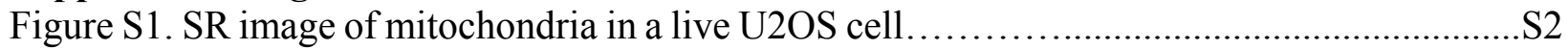

Figure S2. Photophysical characterization and optimization of FAST:HBR-3,5DOM..................S2

Figure S3. Ensconsin-FAST:HBR-3,5DOM in a fixed U2OS cell in PBS..................................S3

Figure S4. ER-Tracker Blue-White DPX for drift correction..................................................... 3

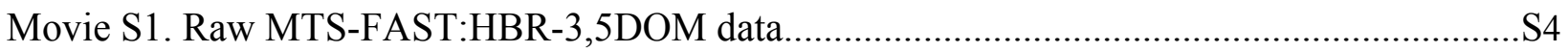




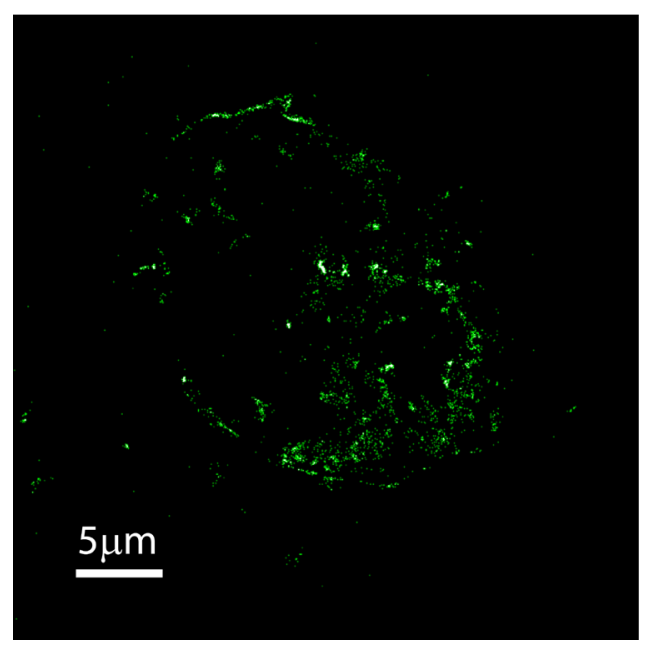

Figure S1. SR image of mitochondria in a live U2OS cell

Rendered super-resolution image of MTS-FAST:HBR-3,5DOM in mitochondria (97 pM HBR3,5DOM, 6515 frames). The same U2OS cell is shown in the conventional fluorescence image in Figure 1A.
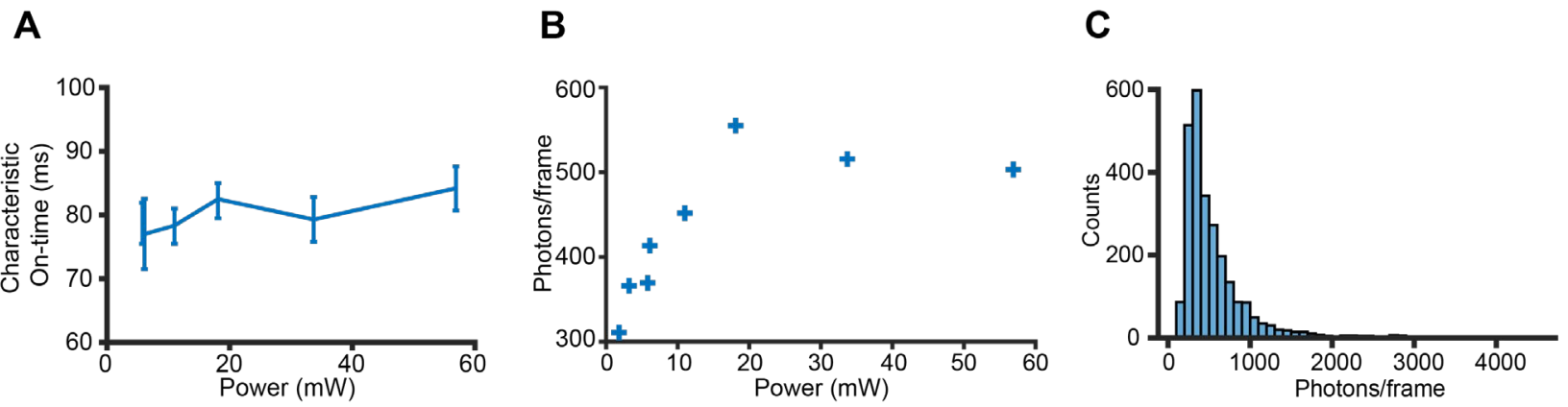

Figure S2. Photophysical characterization and optimization of FAST:HBR-3,5DOM

(A) The characteristic fluorogen on-times (example plot and fit, shown in Figure 2C) are stable over a relevant range of $561 \mathrm{~nm}$ laser powers. Error bars signify $95 \%$ confidence intervals in fitting. (B) Photon counts for different $561 \mathrm{~nm}$ laser power indicate a maximum photon number of 550 photons/frame at $18 \mathrm{~mW}$ (power density $\sim 1 \mathrm{~kW} / \mathrm{cm}^{2}$ ). (C) The distribution of photon counts detected from single FAST-fluorogen complexes at $18 \mathrm{~mW}$. 
A

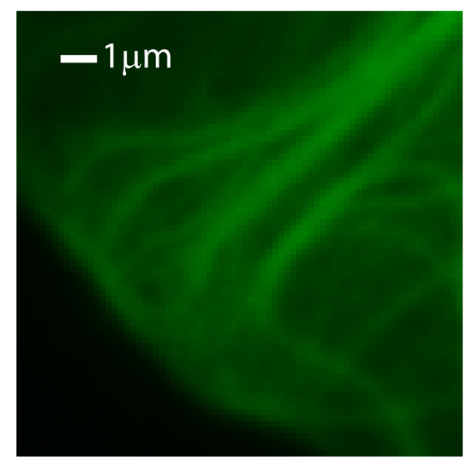

B

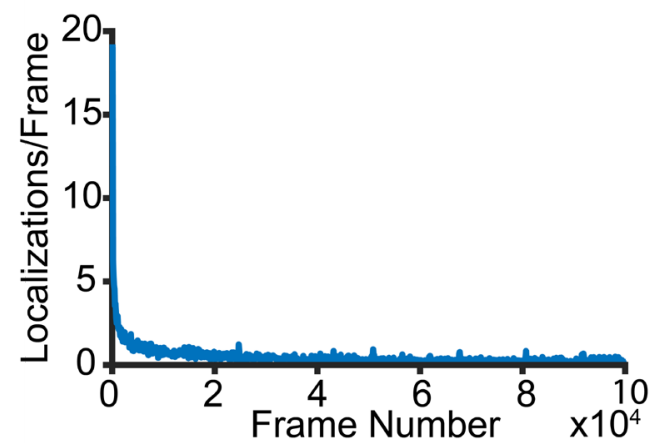

C

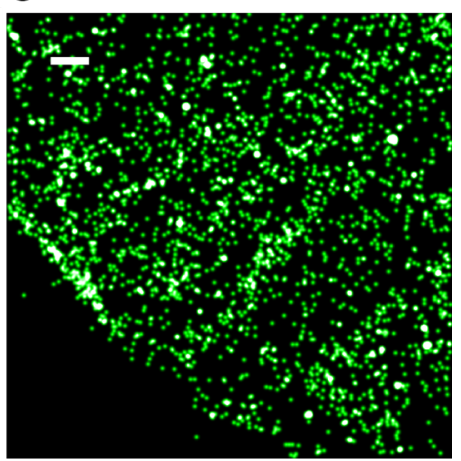

Figure S3. Ensconsin-FAST:HBR-3,5DOM in a fixed U2OS cell in PBS.

(A) Conventional fluorescence image of microtubules (320 nM fluorogen, average 50 frames) confirms specific labeling and proper expression levels of Ensconsin-FAST. (B) The localization density (localizations/frame) significantly decreased over the data acquisition time. (C) Photodestruction of the FAST:fluorogen complex hindered the construction of a high quality superresolution fluorescence image (10 $\mathrm{nM}$ fluorogen, 100000 frames).

A

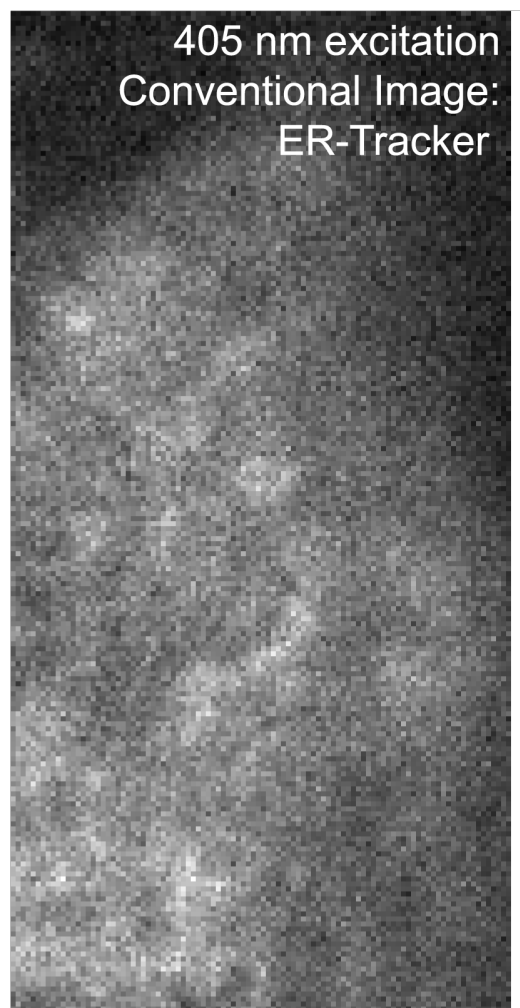

B

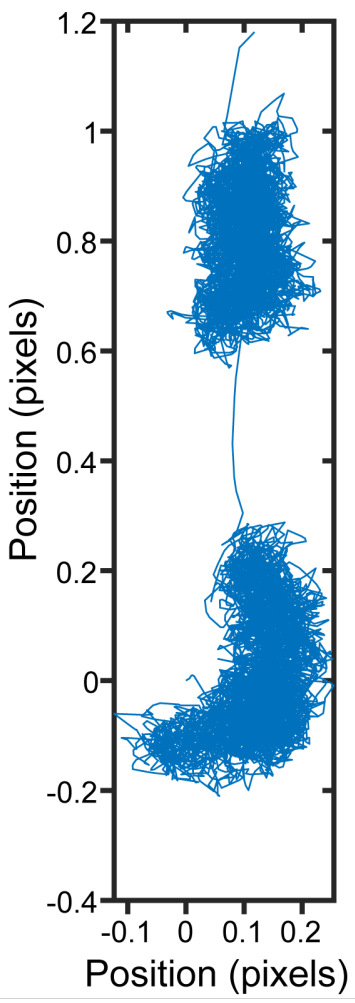

C

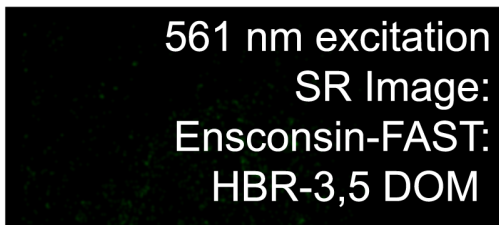
sconsin-FAST:
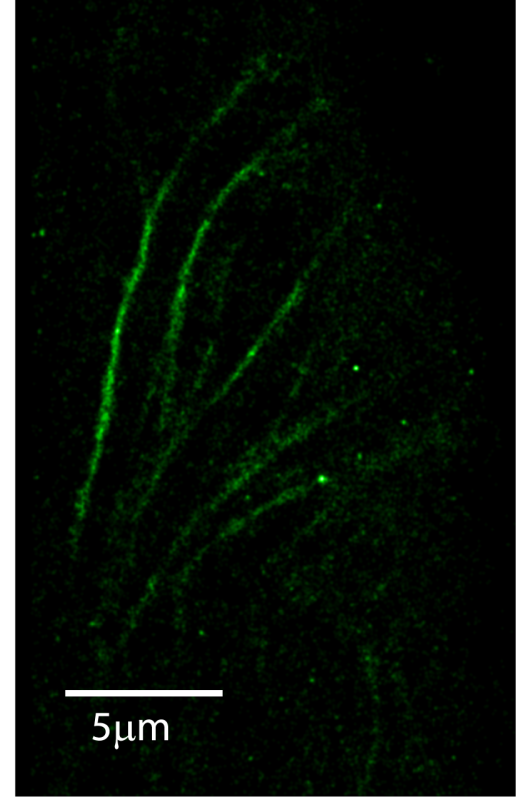

Figures S4. ER-Tracker Blue-White DPX for drift correction

ER-Tracker Blue-White DPX is used in combination with Ensconsin-FAST:HBR-3,5DOM to perform SMLM in fixed U2OS cells. The conventional fluorescence image from the ER-Tracker (A) is used determine the cell sample drift (B) and correct for drift in the rendered SR image (C). 
Movie S1. Raw MTS-FAST:HBR-3,5DOM data

Individual frame of the raw data. The movie showing 1200 representative frames of MTSFAST:HBR-3,5DOM in fixed U2OS cells, plays at 5 fps (acquisition speed, 20Hz). The specific activation of fluorescence allows FAST:fluorogen emitters to be precisely fit and localized over all the frames. 


\section{MATERIALS and METHODS}

\section{Sample Preparation}

All studies were performed using transiently transfected ATCC U2OS cells, that were a kind gift from Dr. Jochen Mueller (University of Minnesota). Cells were maintained in DMEM medium (Gibco) with 10\% fetal bovine serum, and were subcultured in eight-well coverglass chamber slides (Nunc, ThermoFischer) $12 \mathrm{~h}$ before transfection. Transient transfections were performed out using GeneJET (ThermoFisher), according the manufacturer's instructions, 12-24 h prior to the measurements. Immediately before measurement, the growth medium was replaced with Dulbecco's phosphate-buffered saline (PBS) with calcium and magnesium (Gibco). For fixation, cells were treated with $4 \%(\mathrm{v} / \mathrm{v})$ formaldehyde in PBS for $15 \mathrm{~min}$ at room temperature and then were washed three times in PBS. The cells shown in Figure 3 were treated with 100 nM of ERTracker Blue-White DPX (Molecular Probes), according to the manufacturer's instructions, prior to fixation.

The cloning of the Ensconsin-FAST (microtubule-binding protein) and MTS-FAST (mitochondria targeting sequence) plasmids for mammalian cell expression have been previously described. ${ }^{1,2}$

\section{Addition of Fluorogen dyes and GOC}

Stock solutions of HMBR and HBR-3,5DOM were prepared at a concentration of $20 \mathrm{mM}$ in DMSO and stored at $-20^{\circ} \mathrm{C}$. The fluorogen dyes were further diluted and added to the wells at various concentrations prior to measurement. For the typical protein expression levels in the experiments, nanomolar to low micromolar concentrations of the fluorogen resulted in conventional bulk fluorescence, whereas picomolar to low nanomolar concentrations produced a sparse localization density appropriate for single-molecule localization microscopy. For most experiments (except Figure 1F-J and Figure 2E), a fluorogen concentration that resulted in conventional bulk fluorescence was used to select cells and then the overall concentration was diluted for SR imaging with PBS buffer. Alternatively, for the experiment shown in Figure 2E, $100 \mathrm{pM}$ of HBR-3,5DOM was mixed with $25 \mathrm{nM}$ of HMBR (green-yellow fluorogen analog, $\mathrm{Ex} / \mathrm{Em} 488 / 493-797 \mathrm{~nm}$ ) before adding the fluorogens to the well. Cells were selected for measurement using the bulk fluorescence signal from HMBR in the green channel and no dilution was needed prior to super-resolution imaging of HBR-3,5DOM in the red channel. For the experiment shown in Figure 3, glucose oxidase and catalase (GOC) was added to the well just prior to SR imaging at concentrations of $7.1 \mathrm{U} \mathrm{ml}^{-1}$ of glucose oxidase (Aspergillus niger, EMD Millipore), $1 \mathrm{kU} \mathrm{ml}^{-1}$ of Catalase (Aspergillus niger, EMD Millipore), and $4 \mathrm{~g} \mathrm{~L}^{-1}$ of glucose.

\section{Experimental Setup and Data Acquisition}

Super-resolution microscopy experiments were performed on a custom-built microscope, as previously described. ${ }^{3}$ The microscope is based on a Nikon Ti-E inverted microscope with the Perfect Focus System. Three solid state activation/imaging lasers (OBIS 405-100-CW, OBIS 48850-CW, OBIS 561-100-CW; Coherent) are combined using dichroic mirrors, aligned, expanded, and focused to the back focal plane of the oil immersion objective (100 Apo TIRF N.A. 1.49; Nikon). The lasers are controlled directly by the computer. A quad-band dichroic mirror (zt405/488/561/640rpc, Chroma) separates the fluorescence emission from the excitation light. The fluorescence emission light was subsequently split by a $562 \mathrm{~nm}$ beam splitter (T562 lpxr, Chroma) into long (red, $>562 \mathrm{~nm}$ ) and short (green, $<562 \mathrm{~nm}$ ) wavelength channels and filtered by emission bandpass filters (ET595/50 and ET525/50, Chroma). The two channels were 
simultaneously recorded at a frame rate of $20 \mathrm{~Hz}$ with an electron multiplying CCD camera (iXon+ DU897U-CS0-\#BV; Andor). The camera was cooled down to $-68{ }^{\circ} \mathrm{C}$ and the amplifying gain was set to 30 . To estimate the used power density for the activation and excitation laser, we measured the power of the laser at the objective lens and divided it by the illuminated area. The $561 \mathrm{~nm}$ laser powers at the objective ranged from $0.1-1 \mathrm{~mW}\left(\sim 6-62 \mathrm{~W} / \mathrm{cm}^{2}\right)$ for conventional fluorescence imaging and from 15-18 $\mathrm{mW}$ (power density $\sim 1 \mathrm{~kW} / \mathrm{cm}^{2}$ ) for SR imaging. During image acquisition, the axial drift of the microscope stage was stabilized by the Perfect Focus System.

Imaging sequences and powers were varied based on the fluorogen concentration, the cell preparation conditions (fixed versus live), and the experiment. For the experiments shown in Figure 1A-D, Figure 2, and Supplementary Figure 1A, the $561 \mathrm{~nm}$ excitation laser was continuously applied at the power density mentioned above. For Figure 1 F-J, data was recorded with a laser shutter sequence comprising 4 frames of $561 \mathrm{~nm}$ excitation for super-resolution imaging followed by 1 frame of $488 \mathrm{~nm}$ excitation to decrease the fluorescence localization density. The power of the $488 \mathrm{~nm}$ laser started at $\sim 10 \mathrm{~mW}$ and was decreased over time to maintain an optimum localization density (Figure 1G, orange). The SMLM data shown in Figure 3, Figure S3, and Figure S4 was recorded with a laser shutter sequence comprising 9 frames of $561 \mathrm{~nm}$ excitation for SR imaging followed by 1 frame of $405 \mathrm{~nm}$ excitation (power: $23 \mu \mathrm{W}$, density $\sim 1$ $\mathrm{W} / \mathrm{cm}^{2}$ ) to produce a conventional fluorescence ER-Tracker image for sample drift correction. A representative signal from the ER-Tracker is shown in Figure S4A. Both data acquisition and analysis were performed using custom-written software.

\section{Basic Image Analysis}

A typical SR image was generated from a sequence of 50000-100000 image frames, recorded at $20 \mathrm{~Hz}$. For each imaging frame, fluorescent spots were identified and fit to an elliptical Gaussian function to determine their centroid positions, intensities, widths, and ellipticities using INSIGHT software (Zhuang lab, Harvard). Based on these parameters, peaks too dim, too wide, or too elliptical to yield satisfactory localization accuracy were rejected from further analysis. For all localizations the fit parameters such as the $\mathrm{x}$ and $\mathrm{y}$ coordinates, photon number, background photons, and frame of appearance were saved in a list for further analysis. Besides the number of photons detected from each molecule, another factor that limits the localization accuracy was sample drift during the course of the experiment. By correlating the fluorescently labeled ER images recorded in each 10th frame and by tracking the centroid of the correlation function, the sample drift was determined and subtracted from the $\mathrm{x}$ and $\mathrm{y}$ coordinates of all localizations. For image presentation, each localization point was rendered as a normalized 2D Gaussian peak.

Localizations belonging to the same FAST protein will create a spatial signature (radial distribution function, RDF) that defines the uncertainty in position determination and thus the underlying experimental resolution of the system. ${ }^{3-6}$ The pair correlation function (PCF), the distributions of distances between any pair of two localization, was calculated in MATLAB by creating a histogram $(H)$ from the distances between localizations $N$ and by dividing each bin by $N$ and the area at the distance $r_{i}$ :

$$
\operatorname{PCF}\left(r_{i}\right)=\frac{H\left(r_{i}\right)}{N \times\left(\pi\left(r_{i}+\Delta r\right)^{2}-r_{i}^{2}\right)}
$$


where $H_{i}$ is bin $\mathrm{i}$ of the histogram, $r_{i}$ is the distance of bin $i$, and $\Delta r$ is the bin width. The experimental resolution of the FAST:fluorogen system was determined by fitting the PCF with a 2D Gaussian function whose sigma represents the uncertainty in position determination. The resolution profiles in Figure 3 were constructed by adding up the localizations within a defined region and projecting them either onto the $\mathrm{x}$-axis (single localization) or along the axis of the microtubule.

For single molecule diffusion analysis, traces were generated by linking localizations in consecutive frames that appear within a distance of $2 \mu \mathrm{m}$. For each single-molecule trajectory $(n>150)$ longer than three consecutive frames, the mean-square displacement (MSD) was calculated by averaging the displacement of all time intervals with length delta $t$ in a customwritten Igor-Pro program. The diffusion coefficient was calculated by linear fitting of MSD vs lagtime curve and using the $2 \mathrm{D}$ linear diffusion equation: $\left\langle r^{2}\right\rangle=4 D T$. The shaded band surrounding the MSD vs time curve represents a standard error of the mean displacements from the multiple traces. 


\section{REFERENCES:}

(1) Plamont, M.-A., Billon-Denis, E., Maurin, S., Gauron, C., Pimenta, F. M., Specht, C. G., Shi, J., Quérard, J., Pan, B., Rossignol, J., Moncoq, K., Morellet, N., Volovitch, M., Lescop, E., Chen, Y., Triller, A., Vriz, S., Le Saux, T., Jullien, L., and Gautier, A. (2016) Small fluorescence-activating and absorption-shifting tag for tunable protein imaging in vivo. Proc. Natl. Acad. Sci. U. S. A. 113, 497-502.

(2) Li, C., Plamont, M.-A., Sladitschek, H. L., Rodrigues, V., Aujard, I., Neveu, P., Le Saux, T., Jullien, L., and Gautier, A. (2017) Dynamic multicolor protein labeling in living cells. Chem. Sci. $8,5598-5605$.

(3) Puchner, E. M., Walter, J. M., Kasper, R., Huang, B., and Lim, W. A. (2013) Counting molecules in single organelles with superresolution microscopy allows tracking of the endosome maturation trajectory. Proc. Natl. Acad. Sci. U. S. A. 110, 16015-16020.

(4) Veatch, S. L., Machta, B. B., Shelby, S. A., Chiang, E. N., Holowka, D. A., and Baird, B. A. (2012) Correlation functions quantify super-resolution images and estimate apparent clustering due to over-counting. PloS One 7, e31457.

(5) Sengupta, P., Jovanovic-Talisman, T., Skoko, D., Renz, M., Veatch, S. L., and LippincottSchwartz, J. (2011) Probing protein heterogeneity in the plasma membrane using PALM and pair correlation analysis. Nat. Methods 8, 969-975.

(6) Sengupta, P., Jovanovic-Talisman, T., and Lippincott-Schwartz, J. (2013) Quantifying spatial organization in point-localization superresolution images using pair correlation analysis. Nat.

Protoc. 8, 345-354. 